\title{
Fueled by an Epidemic: A Spatial Analysis of Opioid- Positive Drivers Fatally Injured in Motor Vehicle Collisions in West Virginia, 2011-2015
}

\author{
Toni M. Rudisill \\ Injury Control Research Center, West Virginia University, Morgantown, West Virginia, USA \\ *Corresponding author: trudisill@hsc.wvu.edu
}

\begin{abstract}
Background: Narcotics usage is associated with an increased risk of motor vehicle collision and opioid overdose deaths are elevated in West Virginia compared to other states in the United States. This analysis sought to determine the prevalence of narcotics among drivers fatally injured in motor vehicle collisions in West Virginia and to determine if these collisions were clustered in areas of the state where opioid use/abuse is high. Methodology/Principal Findings: Fatal crash data from 2011-2015 were obtained from the Fatality Analysis Reporting System and the locations of the collisions were plotted with spatial software. Spatial analyses, including nearest neighbor indexes, heat maps and hot-spots, were conducted to determine if and where clusters of opioid-positive crashes existed. The results of the spatial analyses were visually compared to the rates of opioid overdose deaths by county, which served as a proxy of opioid use/abuse. Of the 486 drivers, $19 \%(n=94)$ tested positive for opioids. A clustering of opioid positive crashes was detected in the state overall (nearest neighbor index $=0.89$, $\mathrm{p}$-value $=0.055$ ). Hot-spots were detected in the lower regions of the state, which overlapped counties with the highest rates of opioid overdose deaths, and cold-spots were detected in areas with lower opioid overdose death rates. Conclusions/Significance: Individuals using narcotics may still operate motor vehicles, which may pose a threat to all road users in West Virginia. Public health interventions, education, or enforcement may be needed in areas of high opioid use/abuse to raise awareness of driving under the influence of drugs.
\end{abstract}

Keywords: narcotics, automobile driving, fatal, cluster analysis, epidemiology

Cite This Article: Toni M. Rudisill, "Fueled by an Epidemic: A Spatial Analysis of Opioid-Positive Drivers Fatally Injured in Motor Vehicle Collisions in West Virginia, 2011-2015." American Journal of Public Health Research, vol. 5, no. 4 (2017): 124-129. doi: 10.12691/ajphr-5-4-5.

\section{Introduction}

Narcotic usage in the United States (US) has rapidly increased over the past two decades. [1] The morphine milligram equivalents per capita that were prescribed in 2015 were three times higher than those prescribed in 1999. [2] The increase in the consumption of narcotics has not been without consequence. In 2014, nearly 22 million people $\geq 12$ years of age in the US reported having a substance use disorder. [3] Because narcotics are highly addictive, they are often misused and/or abused. Consequently, the prevalence of substance use disorders in the US has increased over $65 \%$ from the 1990's thru the 2000's. [4] A national survey conducted in 2015 found that the prevalence of non-medical use of pain relievers among individuals $\geq 12$ years of age in the US was $2.4 \%$ and $>60 \%$ of these individuals reported misusing prescription opioids. [5] The increase in opioid use has led to a proliferation of emergency department visits, hospitalizations, and deaths. From 2005-2011, emergency department visits due to the non-medical use of opioids increased $117 \%$. [6] Hospitalizations related to opioid use among adults increased over 150\% from 1993 to 2012. [7]
Deaths due to drug overdoses were nearly three times higher in 2014 compared to those in 1999; most of these increases were attributed to opioids. [8] Additionally, many prescription opioid users have switched to more available street drugs, such as heroin or synthetic opioids, to satiate their need. [8]

One state that has been particularly plagued by this drug epidemic is West Virginia. According to the Centers for Disease Control and Prevention, the drug overdose death rate in 2015 was approximately 16 per 100,000 residents nationally, while in West Virginia it was roughly 42 per 100,000 residents. [9] While these figures include all drugs, both prescription and illegal narcotics are largely responsible for these elevated death rates in West Virginia.

To complicate matters, narcotics are one class of drugs which may affect driving ability. Epidemiologic studies have shown that opioid use is associated with an increased risk of motor vehicle collision; however experimental studies have found that driving performance does not vastly differ between those taking opioids versus those receiving placebo. $[10,11,12]$ Studies have shown that as opioid use has increased in the US, more drivers who were fatally injured were detected opioid-positive in postmortem toxicology tests; the rates of opioid-positive 
driver fatalities were nearly three times higher in 2010 compared to 2000. [13]

Although narcotic consumption may increase collision risk and opioid use appears to be elevated in West Virginia, virtually no information exists regarding the relationship between motor vehicle crashes and drivers' opioid use in West Virginia. It is unknown whether this opioid epidemic has impacted fatal motor vehicle collisions in this state. Therefore, the purpose of this study was to determine the prevalence of narcotics among fatally injured West Virginia drivers from 2011-2015; this study also sought to discern if these collisions were clustered in areas of the state that have high rates of opioid use via a spatial analysis.

\section{Materials and Methods}

\subsection{Data Sources}

The primary data source for this analysis was the Fatality Analysis Reporting System (FARS). [14,15] FARS is a publically available database maintained by the National Highway Traffic Safety Administration (NHTSA). All states are required to report collisions to NHTSA when at least one individual involved in a crash on a public roadway dies within 30 days of the incident. The FARS database contains variables pertaining to the crash, vehicles, and individuals involved. Most of these crashes include latitude and longitude coordinates indicating where the collision occurred. The database also lists up to three drug test results and one alcohol test result per individual. The drug test results included in the database are not to include medications administered to the individual as part of post-collision emergency care. [16] While individuals may test positive for more than three drugs, major drug classes are reported in the following priority order: (1) narcotics, (2) depressants, (3) stimulants, (4) marijuana, and (5) other drugs. [17] While drug testing can vary by state, West Virginia performs toxicology tests and reports over $95 \%$ of all fatal crash victims. [16] The FARS data have been described in detail elsewhere. [14,15]

In addition to the FARS data, opioid overdose deaths per county from 2011-2015 were requested and obtained from the West Virginia Health Statistics Center. [18] Population estimates were obtained from the United States Census Bureau to calculate per capita rates for the 5-year period for each county. [19] While drug usage rates are unknown, the opioid overdose death rates served as a proxy of drug use/abuse in the county. A map of West Virginia's counties are included in the Figure 1.

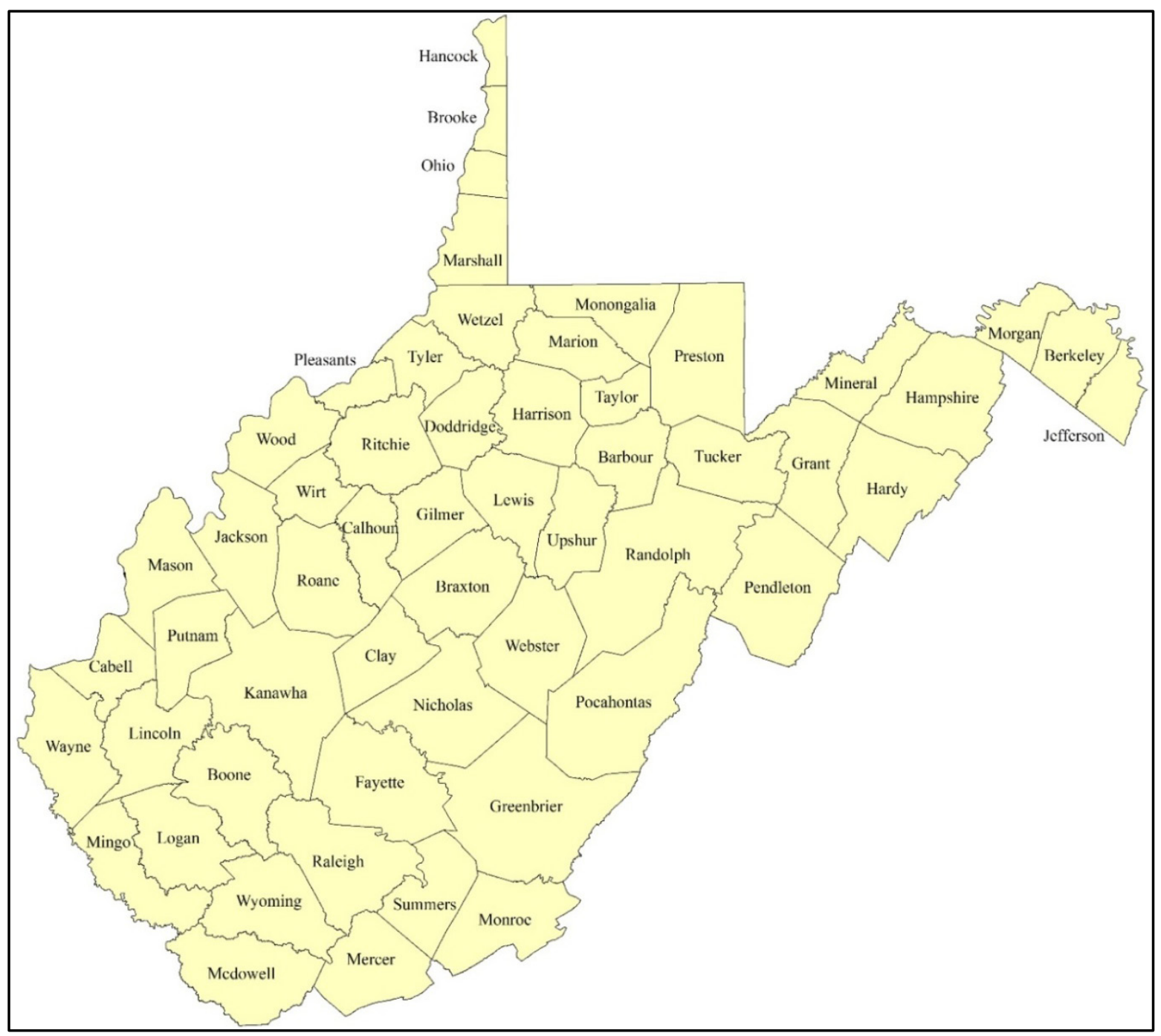

Figure 1. Map of West Virginia Counties 


\subsection{Study Population}

To minimize misclassification, this analysis was limited to all fatally injured drivers of passenger vehicles who died within one hour of the crash. Both time and date of death and of the crash are collected and reported in the FARS data via police, emergency responders, and/or health care providers. Time until death was calculated by subtracting the date and time the person died from their approximate time and date of the crash. While the FARS data are not supposed to include medications administered as post collision care, it was possible that drivers who survived longer than one hour may have been reached by emergency services and administered narcotics to relieve pain after the collision. The collision also had to occur within West Virginia's state boundary between January 1, 2011 and December 31, 2015.

\subsection{Variables}

Drivers' opioid testing status was binary coded; a driver was considered opioid-positive if at least one of their drug test results listed a narcotic or its metabolite. Drivers' age was categorized as $<30,30-59, \geq 60$ years. Driver sex was categorized as male, female, or unknown. Drivers' alcohol testing status was also categorized; drivers were considered alcohol positive if at least a trace or more of alcohol was detected in post-mortem toxicology (i.e. $\geq 0.01 \mathrm{~g} / \mathrm{dl}$ ). Seatbelt usage (shoulder and lap belt, shoulder belt only, or lap belt only) at time of collision was binary coded (i.e. yes or no).

\subsection{Statistical and Spatial Analyses}

Frequencies and percentages of driver demographic characteristics by opioid testing status were calculated using SAS/STAT software (SAS Institute, Cary, NC, USA) version 9.4 .

All collisions meeting the inclusion criteria were plotted with ArcMap software (ESRI, Redlands, CA, USA) version 10.4. An average nearest neighbor index was run on all collisions and opioid-positive collisions to determine if crashes were statistically clustered or dispersed overall in the state. Heat maps were generated for all collisions and opioid-positive collisions to determine and compare crash density. A hot-spot analysis of opioid-positive crashes was then conducted using county as the aggregate factor with the state as the boundary. The hotspot analysis was run to determine if statistically significant hot or cold spots were present among counties. Results of the heat maps and hot-spot analyses were then visually compared to the county opioid overdose death rates to see if opioid-positive crashes were occurring in counties with high overdose rates. The twosided a priori level of significance was 0.10 . This analysis was approved by West Virginia University's Institutional Review Board (protocol \#1704562617). The data were collected and analyzed in 2017.

\section{Results}

From 2011-2015, there were 486 drivers who met the study inclusion criteria (Table 1). Of these drivers, 19.3\% $(\mathrm{N}=94)$ tested positive for opioids. The majority of drivers were male $(72 \%)$ and aged $30-59$ years $(47 \%)$. Most of these drivers tested negative for alcohol $(67 \%)$, but were not wearing a seat belt (65\%) at time of collision. Only $26 \%$ of opioid-positive drivers were wearing a seat belt at time of collision compared to $38 \%$ of opioid-negative drivers.

Table 1. West Virginia drivers fatally injured in a motor vehicle collision by opioid testing status, 2011-2015 $(\mathrm{N}=486)^{\mathrm{a}}$

Opioid Negative Opioid Positive Total

\begin{tabular}{lllllll} 
Characteristic & $\mathrm{N}$ & $\%$ & $\mathrm{~N}$ & $\%$ & $\mathrm{~N}$ & $\%$ \\
\hline
\end{tabular}

Sex

$\begin{array}{rllllll}\text { Male } & 283 & 72.2 & 66 & 70.2 & 349 & 72.0 \\ \text { Female } & 109 & 27.8 & 28 & 29.8 & 137 & 28.0 \\ \text { Unknown } & 0 & & 0 & & 0 & \end{array}$

Age (in years)

$\begin{array}{rllllll}<30 & 123 & 31.4 & 30 & 31.9 & 153 & 31.5 \\ 30-59 & 173 & 44.1 & 53 & 56.4 & 226 & 46.5 \\ \geq 60 & 96 & 24.5 & 11 & 11.7 & 107 & 22.0 \\ \text { Unknown } & 0 & & 0 & & 0 & \end{array}$

Alcohol

$\begin{array}{ccccccc}\text { Positive } & 128 & 33.7 & 30 & 31.9 & 158 & 33.3 \\ \text { Negative } & 252 & 66.3 & 64 & 68.1 & 316 & 66.7 \\ \text { Unknown } & 12 & & 0 & & 12 & \end{array}$

Seat belt use

$\begin{array}{rllllll}\text { Yes } & 129 & 37.8 & 22 & 26.2 & 151 & 35.5 \\ \text { No } & 212 & 62.2 & 62 & 73.8 & 274 & 64.5 \\ \text { Unknown } & 51 & & 10 & & 61 & \end{array}$

a: Percentages may not equal $100 \%$ due to rounding.

The average nearest neighbor index (NNI) for all crashes $(\mathrm{NNI}=0.75, \mathrm{Z}$-score $=-10.55, \mathrm{p}$-value $=<0.0001)$ and for opioid-positive crashes $(\mathrm{NNI}=0.89, \mathrm{z}$-score $=-1.92$, $\mathrm{p}$-value $=0.055)$ indicated that these collisions were clustered in the state overall (not shown). Figure 2 shows the locations of all crashes (left panel) and those crashes where the driver was opioid-positive (right panel). For all driver fatalities, collisions were condensed around the state's urban centers such as Morgantown, Charleston, and Martinsburg (Figure 3, left panel). For opioid-positive collisions, these were found around Fairmont and Martinsburg, but most were clustered towards the southern region of West Virginia (Figure 3, right panel). The hot-spot analysis also showed statistically significant clusters in the southern counties of the state (Figure 4, top panel). The hot-spots mainly overlapped counties with high rates of opioid overdose death rates, while cold-spots generally overlapped counties with low opioid overdose death rates (Figure 4, lower panel). There were a few exceptions to this trend, which included Hancock, Brooke, Wood, Berkley, Morgan, and Harrison counties. 


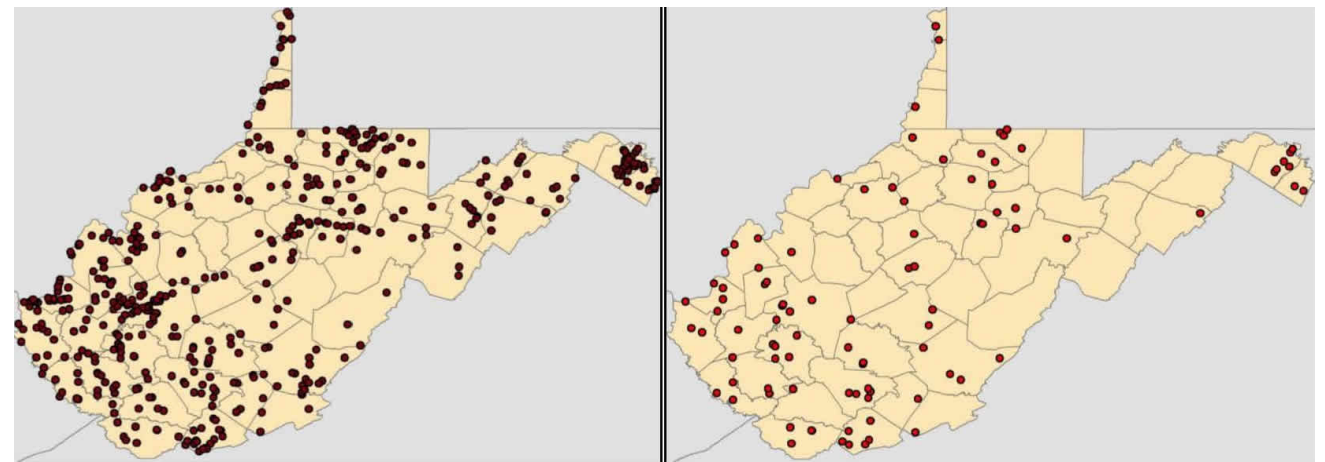

The left panel shows locations of all collisions, whereas the right panel shows collisions in which the driver tested positive for narcotics in post-mortem toxicology.

Figure 2. Locations of collisions in West Virginia from 2011-2015

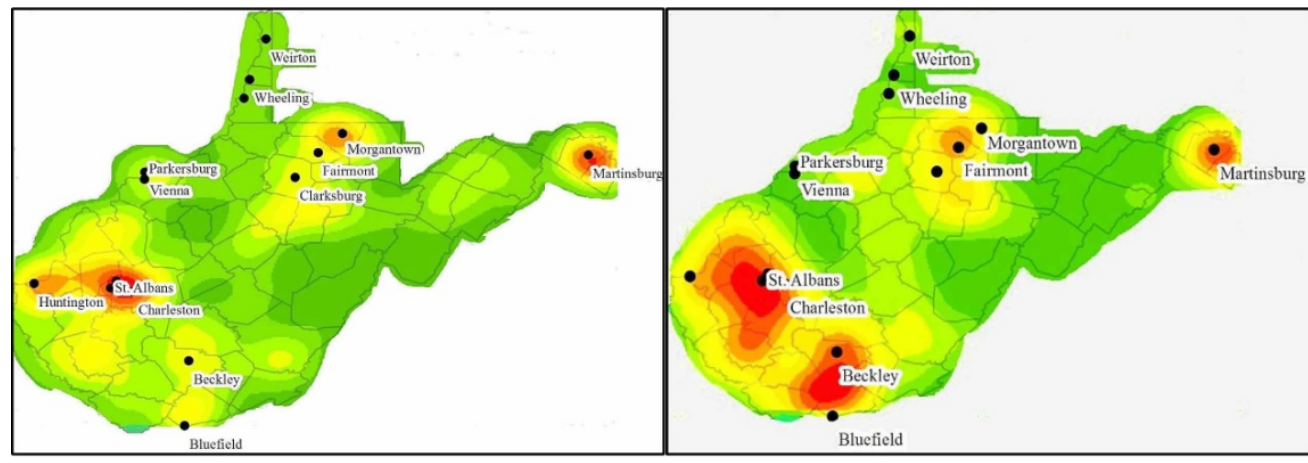

The left panel shows the density of all crashes, whereas the right panel shows the density of opioid-involved crashes.

Figure 3. Heat maps showing density of fatal collisions

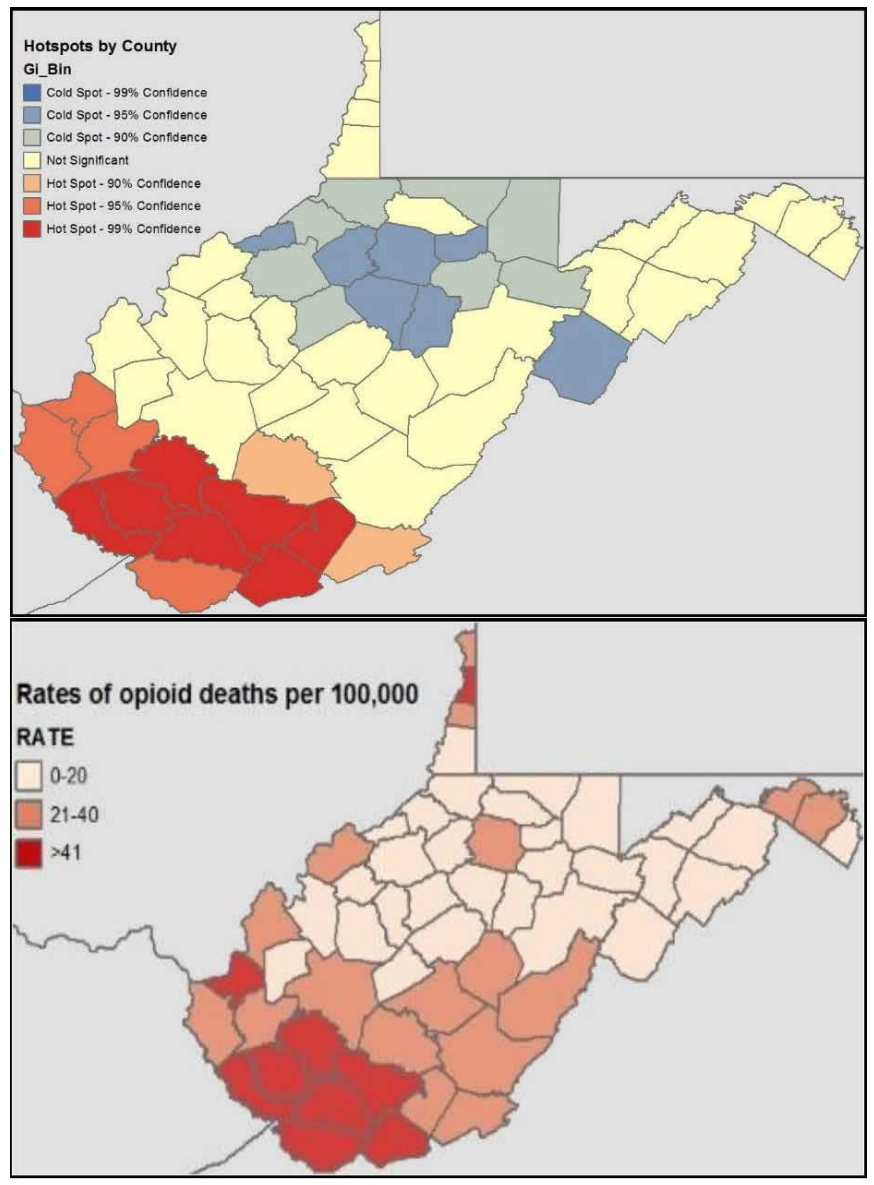

The top panel shows the results of the hot spot analysis, whereas the lower panel is the rates of opioid overdose deaths per 100,000.

Figure 4. Comparison of county-level hot spot analysis to rates of drug overdose deaths by county 


\section{Discussion}

The findings of this analysis indicate that the opioid epidemic in West Virginia may have influenced drivers. Fatally injured drivers who tested positive for opioids tended to experience their collision in areas of the state known to have high rates of opioid overdose deaths, whereas few collisions were observed in areas known to have low opioid overdose death rates. This relationship was not likely driven by population because the patterns between all crashes and opioid-positive collisions occurred in slightly different areas. Fatal collisions, in general, tended to cluster around urban centers. Collisions would naturally be more concentrated in these areas with more people commuting to work, school, etc. However, the patterns for opioid-positive collisions were heavily centered toward the lower counties in the state, which are less populated. Therefore, these findings could suggest that individuals may be more likely to drive under the influence of opioids in areas of high opioid use/abuse.

Interestingly, this analysis also found that seatbelt usage among opioid-positive drivers was very low. Research indicates that nearly $88 \%$ of all US drivers typically wear safety belts when operating a motor vehicle. [20] However, studies also show that seat belt usage rates are significantly lower in Appalachian states, such as West Virginia. [21] Seatbelts can reduce the amount of injuries and fatalities sustained in motor vehicle collisions by nearly 50\%. [22] It is quite possible that if these drivers were wearing safety belts that they may have survived their collision.

These findings have important public health and traffic safety ramifications for those travelling in West Virginia. Epidemiologic studies have found that narcotic usage is associated with increased risk of motor vehicle collision and that the risk of collision increases with dosage. [10,11,12] It is possible that drivers using these substances could be negatively affected by these substances and possibly crash. This could pose a threat not only to the opioid-using driver, but to other road users, such as passengers, pedestrians, or pedalcyclists. This study may also highlight the need for intervention. It is unknown whether the narcotics consumed by these drivers were prescriptions or illicit drugs, such as heroin, or whether these drugs were being misused/abused by the driver. However, it is possible that those taking these drugs were unaware that their collision risk may have been elevated. Public health interventions, driver education, or enforcement efforts pertaining to driving under the influence of drugs, and even seat belt usage, may be needed in areas of high opioid use/abuse. While specific interventions pertaining to drug use while driving are lacking in the extant literature, it appears that interventions focusing driver education may potentially be beneficial. [23] Healthcare providers treating individuals with substance use disorders or writing prescriptions for narcotics may also need to remind patients about the potential increased risk of motor vehicle collision that opioids, including buprenorphine and methadone, may pose.

\subsection{Limitations}

While the results of this study are informative, they are not without limitation. First, this study did not examine which specific drugs were consumed by these drivers. This was not investigated because opioids are often rapidly metabolized. Therefore, it may not have been possible to determine the parent drug if only the metabolite was identified in toxicological testing. Second, the FARS data are qualitative and do not include the concentration of the drug identified. Therefore, quantitative measures could not be explored. Third, this study focused strictly on West Virginia. Especially in the instances of illegal drug trafficking, surrounding states may have influenced the trends seen in West Virginia. If larger areas or more states were analyzed in aggregate, the clustering observed in this analysis may have disappeared or intensified; modifiable area unit problems are a well-known and inherent limitation of spatial analyses. Fourth, this analysis focused on where the crash occurred, not on where the driver resided. As driver residence was unknown, this relationship could not be explored. Fifth, there is no one true measure for drug use/abuse in an area. This study used drug overdose death rate as a proxy for this drug use/abuse. Drug use/abuse in an area could be assessed by drug arrests, retail drug sales, narcotics-related hospitalizations, etc. Additionally, this study was spatial in nature; therefore, it could not determine causality. This study does not prove that these drivers abused or misused these drugs, engaged in illegal drug activity, or that their narcotic usage caused their collision. Lastly, this study looked simply at whether narcotics-related crashes were clustered in areas with high rates of drug use/abuse. Fatal collisions can result from a plethora of factors. Thus, there are potential confounding factors that cannot be accounted. For example, the driving habits of individuals who use narcotics vs. those who do not are unknown. Drivers who use narcotics could have unsafe driving habits or spend differing times at risk of collision by driving more or less miles than non-users. Also, the type of driving environment that narcotics users may normally drive could influence the pattern, such as the type of road (multi-lane highway, rural roads, etc.), road curvature, road condition, traffic congestion, or time of day. More research would be needed to elucidate these differences.

\section{Conclusion}

From 2011-2015, nearly one in five drivers fatally injured in West Virginia tested positive for narcotics. These collisions tended to be clustered in areas of high opioid use/abuse and less frequent in areas of low opioid use/abuse. Because narcotics are associated with an increased risk of motor vehicle collision, those taking opioids may pose a risk not only to themselves, but also to other road users. Targeted public health interventions, driver education, or enforcement may be needed in areas known to have high rates of opioid use/abuse. Interventions concerning seat belt usage may also be warranted in these areas as so few opioid-positive drivers were wearing safety belts at time of their collision.

\section{Acknowledgments}

TMR received salary support from Centers for Disease Control and Prevention grant R49 CE002109. The study 
sponsors did not have any role in the study design, collection, analysis, interpretation of data, writing of the report, or the decision to submit the report for publication.

\section{Statement of Competing Interests}

The author has no competing interests.

\section{List of Abbreviations}

FARS=Fatality Analysis Reporting System;

NHTSA=National Highway Traffic Safety Administration; $\mathrm{NNI}=$ nearest neighbor index;

$\mathrm{US}=$ United States;

\section{References}

[1] Schuchat, A., Houry, D., Guy, G.P., Jr, "New data on opioid use and prescribing in the United States", JAMA, 318 (5), 425-426, 2017.

[2] Guy, G.P., Jr., Zhang, K., Bohm, M.K., et al, "Vital Signs: Changes in Opioid Prescribing in the United States, 2006-2015", MMWR, 66 (26), 697-704, 2017.

[3] Lipari, R., Van Horn, S., "Trends in substance use disorders among adults aged 18 or older", 2017, [Online] Available: https://www.samhsa.gov/data/sites/default/files/report_2790/Short Report-2790.html. [Accessed Aug. 1,] 2017.

[4] Blanco, C., Alderson, D., Ogburn, E., et al, "Changes in the prevalence of non-medical prescription drug use and drug use disorders in the United States: 1991-1992 and 2001-2002", Drug and Alcohol Depend, 90 (2-3), 252-260, 2007.

[5] Center for Behavioral Health Statistics and Quality, "Key substance use and mental health indicators in the United States: Results from the 2015 national survey on drug use and health", 2016, [Online] Available:

https://www.samhsa.gov/data/sites/default/files/NSDUH-FFR12015/NSDUH-FFR1-2015/NSDUH-FFR1-2015.pdf. [Accessed July, 1 2017].

[6] Crane, E., "Emergency department visits involving narcotic pain relievers", 2015, [Online] Available:

https://www.samhsa.gov/data/sites/default/files/report_2083/Short Report-2083.html. [Accessed July 1, 2017].

[7] Owens, P., Barrett, M., Weiss, A., Washington, R., Kronick, R. "Hospital inpatient utilization related to opioid overuse among adults, 1993-2012", 2014, [Online] Available: https://www.hcupus.ahrq.gov/reports/statbriefs/sb177-Hospitalizations-for-OpioidOveruse.pdf. [Accessed July 1, 2017].

[8] Rudd, R.A., Aleshire, N., Zibbell, J.E., Gladden, R.M., "Increases in Drug and Opioid Overdose Deaths--United States, 2000-2014", MMWR, 64 (50-51), 1378-1382, 2016.
[9] Centers for Disease Control and Prevention, "WISQARS", 2017, [Online] Available: https://www.cdc.gov/injury/wisqars/. [Accessed Apr. 25, 2017].

[10] Morland, J., "Driving under the influence of non-alcohol drugs", Forensic SciRev, 12, 80-105, 2000.

[11] Gjerde, H., Strand, M.C., Morland, J., "Driving Under the Influence of Non-Alcohol Drugs--An Update Part I: Epidemiological Studies", Forensic Sci Rev, 27 (2), 89-113, 2015.

[12] Strand, M.C., Gjerde, H., Morland, J., "Driving under the influence of non-alcohol drugs--An update. Part II: Experimental studies", Forensic Sci Rev, 28 (2), 79-101, 2016.

[13] Rudisill, T.M., Zhao, S., Abate, M.A., Coben, J.H., Zhu, M., "Trends in drug use among drivers killed in U.S. traffic crashes, 1999-2010", Accid Anal Prev, 70, 178-187, 2014.

[14] National Highway Traffic Safety Administration, "Fatality Analysis Reporting System Analytical Users Manual 1975-2015", 2016, [Online] Available:

https://crashstats.nhtsa.dot.gov/Api/Public/Publication/812315. [Accessed July 26, 2016].

[15] National Highway Traffic Safety Administration, "Fatality Analysis Reporting System (FARS)", 2017, [Online] Available: https://www.nhtsa.gov/research-data/fatality-analysis-reportingsystem-fars. [Accessed Jan. 3, 2017].

[16] National Highway Traffic Safety Administration, "Drug Involvement of Fatally Injured Drivers", 2010, [Online] Available: www-nrd.nhtsa.dot.gov/Pubs/811415.pdf. [Accessed Jan. 3, 2015]

[17] Centers for Disease Control and Prevention, "Alcohol and other drug use among victims of motor vehicle crashes-West Virginia 2004-2005”. MMWR, 55 (48), 1293-1296, 2006.

[18] West Virginia Department of Health and Human Resources, "Health Statistics Center", 2017, [Online] Available: http://www.wvdhhr.org/bph/hsc/. [Accessed Feb. 3, 2017].

[19] United States Census Bureau, "Population and housing unit estimates", 2017, [Online] Available:

https://www.census.gov/programs-surveys/popest.html. [Accessed Apr. 27, 2017].

[20] National Highway Traffic Safety Administration, "Seat belt use in 2015-overall results", 2016, [Online] Available: https://crashstats.nhtsa.dot.gov/Api/Public/ViewPublication/81224 3. [Accessed Apr. 27, 2017].

[21] Birru, H., Rudisill, T.M., Fabio, A., Zhu, M., "A comparison of self-reported seat belt usage among the Appalachian and nonAppalachian United States", Ann Epidemiol, 26 (3), 227-230, 2016.

[22] National Highway Traffic Safety Administration, "Final regulatory impact analysis amendment to Federal Motor Vehicle Safety Standard 208 passenger car front seat occupation protection", 1984, [Online] Available: http://www-nrd.nhtsa.dot.gov/pubs/806572. [Accessed Apr. 4, 2015].

[23] Miller, P.G., Curtis, A., Sønderlund, A., Day, A., Droste, N, "Effectiveness of interventions for convicted DUI offenders in reducing recidivism: a systematic review of the peer-reviewed scientific literature", Am J Drug Alcohol Abuse, 41 (1), 16-29, 2015. 